\title{
Solução M-Health no pré-diagnóstico da Laringe
}

\author{
Alternative Title: M-Health Solution on pre-diagnosis of larynx \\ Estefânia Mayumi Fuzyi \\ Londrina State University, \\ Rod. Celso Garcia Cid km 380 \\ Londrina, Paraná \\ emfuzyi@gmail.com \\ Matheus Camilo da Silva \\ Londrina State University, \\ Rod. Celso Garcia Cid km 380 \\ Londrina, Paraná \\ matheuscmilo@gmail.com \\ Sylvio Barbon Jr \\ Londrina State University, \\ Rod. Celso Garcia Cid km 380 \\ Londrina, Paraná \\ barbon@uel.br
}

\begin{abstract}
RESUMO
O estudo de novas abordagens que buscam aperfeiçoar o diagnóstico de patologias nas pregas vocais, é um dos principais motivadores para pesquisa em saúde baseado na voz. Não somente pela criação de novas técnicas, mas também no uso de tecnologias existentes com novas abordagens, tais como as tecnologias móveis em campos em que elas não foram exploradas, ou não foram exploradas completamente. Este artigo tem como objetivo apresentar uma ferramenta m-health para o diagnóstico precoce de doenças nas pregas vocais por meio de análises da frequência fundamental da voz do indivíduo juntamente com características dos hábitos do paciente. Assim, pretende-se melhorar a qualidade do diagnóstico, acelerando o processo e acrescentando outras contribuições de um m-health como o suporte às decisões médicas. Para avaliar os resultados obtidos no diagnóstico automatizado via ferramenta, foi utilizado uma base de vozes de pacientes de ambos os gêneros, já diagnosticados como patológicos. Atingiu-se um resultado de $95 \%$ de acerto, considerando uma diferença de $10 \mathrm{~Hz}$.
\end{abstract}

\section{Palavras-Chave}

Prontuário eletrônico, M-health, Voz, Laringe, Aplicativo Móvel

\begin{abstract}
The study of new approaches that seek to improve the diagnosis of pathologies in the vocal folds process, is one of the main motivators for health research based on voice. Not only by the creation of new techniques, but in the use of already existing technologies using new approachs, such as the mobile technologies in fields they were never fully explored, or barely explored at all. This article is intended to further increase the development of the m-health's field, specifically the early diagnosis of the vocal fold's diseases through analysis of the fundamental frequency of the speaker's vocalization improving the support in medical decision. The result shows $95 \%$ with a minimum of $10 \mathrm{hz}$ difference.
\end{abstract}

Permission to make digital or hard copies of all or part of this work for personal or classroom use is granted without fee provided that copies are not made or distributed for profit or commercial advantage and that copies bear this notice and the full citation on the first page. To copy otherwise, to republish, to post on servers or to redistribute to lists, requires prior specific permission and/or a fee.

SBSI 2015, May 26th-29th, 2015, Goiânia, Goiás, Brazil

Copyright SBC 2015.

\section{Categories and Subject Descriptors}

J.3 [Life and Medical Sciences]: Medical information systems; K.4.1 [Computers and Society]: Public Policy Issues - Computer-related health issues; C.3 [Specialpurpose and Application-based Systems]: Signal processing systems

\section{General Terms}

Experimentation, Design

\section{Keywords}

Electronic Medical Record, M-Health, Voice, Larinx, Mobile Application

\section{INTRODUÇÃO}

M-Health ou m-health, é uma abreviação do termo Mobile Health (saúde móvel), ou seja, trata-se do uso de aparelhos móveis (smartphones, tablets, dispositivos com sensores e seus sistemas) em prol da saúde [1]. Consequentemente, possui as mesmas vantagens das tecnologias móveis, como o acesso remoto e simultâneo, baixo custo monetário [5] e integração com outros sistemas de informação.

O crescente interesse no potencial das tecnologias móveis em auxiliar e aperfeiçoar o atendimento na área da saúde, leva à gradual propagação e avanço no campo de m-health, principalmente em países emergentes, os quais possuem maiores necessidades no uso de tecnologias de baixo custo em seus sistemas de saúde [15]. O m-health pode ser de grande contribuição para os atendimentos médicos em uma diversidade de formas, entretanto, a forma mais importante, é a possibilidade de manuseio de dados e informações médicas em tecnologias móveis, de lugares remotos e entre diferentes profissionais da área da saúde.

Todo atendimento na área da saúde requer e gera informação, para que um profissional possa atender adequadamente o paciente. Além de registros das atividades médicas atuais, é necessário o histórico do paciente, inclusive de diferentes áreas da saúde, que dêem suporte às suas decisões de diagnóstico e/ou tratamento [2]. Esses registros devem estar disponíveis no suporte à decisões em atendimentos futuros ou paralelos. O prontuário médico é o conjunto de documentos padronizados e ordenados, em que são armazenadas as informações clínicas e administrativas do indivíduo ao longo de sua vida.

O prontuário eletrônico, nada mais é do que a versão digital do prontuário médico comum, que, quando combinado 
com dispositivos móveis, permite-se ser utilizado como mhealth, e portanto, herdando todos os benefícios do campo. É uma ferramenta moderna, de extrema importância no cuidado médico, que traz agilidade e eficiência no acesso, registro e compartilhamento de informação entre profissionais da área da saúde [5].

Dentre as diversas especialidades médicas, existe a Laringologia, uma área da Otorrinolaringologia que compreende as doenças da laringe e da voz. Nessa, como na maioria das áreas, um diagnóstico precoce pode ser fundamental no tratamento com sucesso de uma doença, como o câncer de laringe, que em estados iniciais tem uma possibilidade de cura relativamente alta [22].

A produção da voz ocorre na laringe, por meio da passagem do ar expirado por entre as pregas vocais. A alteração da produção de voz é chamada de disfonia, seu reconhecimento e análise pode ser realizada pela sustentação da vogal $/ a /[21]$ por exemplo, por meio de técnicas de processamento digital de sinais, como Janelamento e a Transformada Rápida de Fourier (Fast Fourier Transform - FFT) [4]. A FFT proporciona a avaliação no domínio da frequência da vocalização, propiciando uma alternativa não-invasiva de custo inferior a exames convencionais para diagnóstico de doenças na laringe [4].

A análise da frequência de voz quantificada pela FFT por meio da vocalização pode ser realizada por um software, sendo possível ser implementada junto a um prontuário eletrônico. Desta forma, ao reunir possíveis informações acústicas auxiliares sobre a saúde da voz do paciente durante a anamnese, é possível auxiliar profissionais das áreas da Otorrinolaringologia e Fonoaudiologia na decisão médica.

Atualmente, a maioria dos diagnósticos de distúrbios na região laríngea para doenças como, por exemplo, a Distonia Laríngea, é feito a partir da coleta de imagens da região da laringe. Estes exames possuem alto custo [6], além de serem invasivos e de difícil realização em pacientes dentro da faixa etária pediátrica [19]. Exames como a videolaringoestroboscopia, no caso da Distonia Laríngea, em que uma micro câmera acoplada a uma fonte de luz e ligada a um aparelho de monitoramento é inserida na laringe através da narina ou pela garganta, o que leva em alguns casos a intenso reflexo nauseante, tornando difícil e, algumas vezes, impossível a realização do exame [12].

Portanto, existe a necessidade de avanços tecnológicos nos métodos de diagnóstico de doenças na região laríngea, avanços que possam substituir os métodos convencionais, que são caros e de difícil realização. Métodos como o m-health, que são não-invasivos, rápidos e automatizados, podem auxiliar o especialista na conclusão de um pré-diagnóstico, e, dependendo do resultado, poupar o paciente de possíveis exames desnecessários.

Para um diagnóstico preciso, o profissional da área da saúde necessita, além de um exame, estabelecer um histórico médico do paciente por meio de informações do prontuário do mesmo. Este trabalho tem como objetivo apresentar uma solução m-health para apoio à decisão médica na avaliação da saúde vocal em ambientes remotos, facilitando o encaminhamento e pré-diagnóstico, que possua tanto um prontuário eletrônico com dados e informações médicas e administrativas do paciente, quanto uma ferramenta computacional capaz de análise de voz.

Este documento está estruturado como segue, a Seção 2 apresenta trabalhos relacionados com o trabalho desenvol- vido, a Seção 3 explica a proposta e a funcionalidade da solução m-health desenvolvida neste trabalho. A Seção 4 discorre sobre os conceitos, materiais e métodos que possibilitaram a criação da solução m-health. A Seção 5 apresenta possíveis discussões sobre os resultados deste trabalho, que comprovam sua eficiência. A Seção 6 destaca as conclusões finais obtidas através do trabalho, além da apresentação de possíveis trabalhos futuros.

\section{TRABALHOS RELACIONADOS}

Através de logs de transações do sistema do prontuário eletrônico do paciente, utilizado no estudo, [18] pôde verificar o uso do mesmo pelos médicos. Com os resultados obtidos, foi possível constatar grande interesse dos médicos selecionados em utilizar um prontuário eletrônico, sendo que $70 \%$ deles consultaram o histórico de vida do paciente, $100 \%$ leram as consultas anteriores e cadastraram pelo menos um Código Internacional da Doença em cada consulta. Além da melhoria na gestão da saúde e da informação, por auxiliar na medicina preventiva, por exemplo, devido a constante atualização dos dados do paciente.

$[17,13]$ apresentam estudos de casos de implantação e utilização de prontuários eletrônicos de pacientes em hospitais e complexos hospitalares, que demonstraram grande aceitação pelos usuários do sistema e demonstrou melhoria na qualidade de informação e no atendimento aos pacientes.

Na revisão de literatura realizada em [14], com período de 2005 à 2011, diversos artigos evidenciaram a importância da implantação dos prontuários eletrônicos, porém também discutem a resistência dos funcionários, o alto custo de investimento em hardware e software e o risco da perda de contato entre médico e paciente.

Em [11], foi realizado um levantamento de artigos, no período de 2005 à 2010 , de trabalhos científicos que utilizaram softwares, seja para avaliação ou na terapia fonoaudiológica. Ele constatou um crescimento médio de $27 \%$ de artigos publicados, nas revistas pesquisadas, e de $89 \%$ de artigos que utilizam software.

Para o reconhecimento automatizado de distonia na laringe, [20] utiliza a sustentação do pitch. No desenvolvimento da ferramenta, foi aplicada a Transformada de Fourier para a obter o sinal no domínio da frequência, o que possibilita encontrar o valor do pitch e realizar a sua avaliação. Para validar os resultados obtidos, eles foram comparados com avaliações de especialistas e houve a conclusão que os resultados são equivalentes.

Dessa forma, a ferramenta proposta visa combinar o prontuário eletrônico e suas vantagens, com uma solução que avalia o sinal de voz do paciente e calcula o valor do pitch, o que possibilita a realização do pré-diagnóstico por um especialista, além de ser desenvolvida para dispositivos móveis. Portanto, não apresenta um alto investimento por parte das instituições e não tem o objetivo de eliminar a interação entre médico e paciente.

\section{PROPOSTA DE M-HEALTH}

Neste trabalho é apresentada uma solução m-health para realizar o pré-diagnóstico da Laringe. A proposta é unir o prontuário eletrônico e a análise de vocalização em uma aplicação voltada para aparelhos móveis.

Nela, o usuário poderá inserir informações do paciente referentes ao prontuário, como dados pessoais: "nome", "pro- 
fissão" e "tipo de necessidades especiais", por exemplo, e dados complementares como presença de rouquidão, garganta seca, entre outros. Além da gravação da voz do paciente e sua avaliação automatizada.

As telas e campos do cadastro do prontuário eletrônico da ferramenta foram desenvolvidas como em Marques et al. [16]. Marques et al. [16] explicita sobre quais tipos de dados são relevantes e quais campos devem constar em um prontuário eletrônico com foco em análises da voz, afim de compilar informações suficientes para a criação de um histórico médico adequado.

A aplicação conta com seis funcionalidades básicas no menu inicial, como mostrado na Figura 1, das quais, "Novo Cadastro", "Pesquisa", "Análise da voz", "Anamnese para diagnóstico", "Dados Complementares", e, "Hábitos vocais", possuem funcionalidades de prontuário eletrônico, ou seja, sua função é coletar dados sobre o paciente. Enquanto "Gravação" e "Resultados" possuem as funcionalidades de gravação e análise da vocalização.

\begin{tabular}{|c|c|}
\hline $\begin{array}{c}\text { Prontuário de Pacientes } \\
\text { Novo Cadastro }\end{array}$ & Pesquisa \\
\hline Análise da Voz & Anamnese para Diagnóstico \\
\hline Dados Complementares & Hábitos Vocais \\
\hline Gravação & Resultado \\
\hline
\end{tabular}

Figura 1: Menu da aplicação

A funcionalidade "Novo Cadastro", permite ao usuário o cadastro de informações gerais de um paciente no banco de dados da aplicação, como "nome", "gênero" e "RG", afim de identificá-lo. Em seguida, pode-se obter informações de pacientes registrados no banco de dados por meio da funcionalidade "Pesquisa". "Análise da voz", permite o registro de dados referentes à qualidade de voz de um paciente, anterior e posteriormente à uma prova física aplicada por um profissional, como a prova de indução à fadiga vocal [16], contribuindo para a constituição do pré-diagnóstico.

As funcionalidades "Anamnese para diagnóstico", "Dados complementares" e "Hábitos Vocais", permitem ao usuário o registro de dados médicos referentes à saúde vocal de um paciente no banco de dados da solução m-health, contribuindo para a formação de um histórico médico. A aplicação também possibilita a gravação da vocalização de um paciente na funcionalidade "Gravação" e a análise automatizada da frequência fundamental dessa gravação, além da apresentação do resultado na funcionalidade "Resultado".

Existem diferentes campos de preenchimento na solução m-health proposta, de acordo com o tipo de informação que deverá ser registrado, variando entre simples campos de texto para preenchimento de informações como "nome" e "endereço", como mostra a Figura 2. Para outros tipos de informação são utilizadas barras de rolagem, por exemplo, para o preenchimento de informações como nível de rouqui-

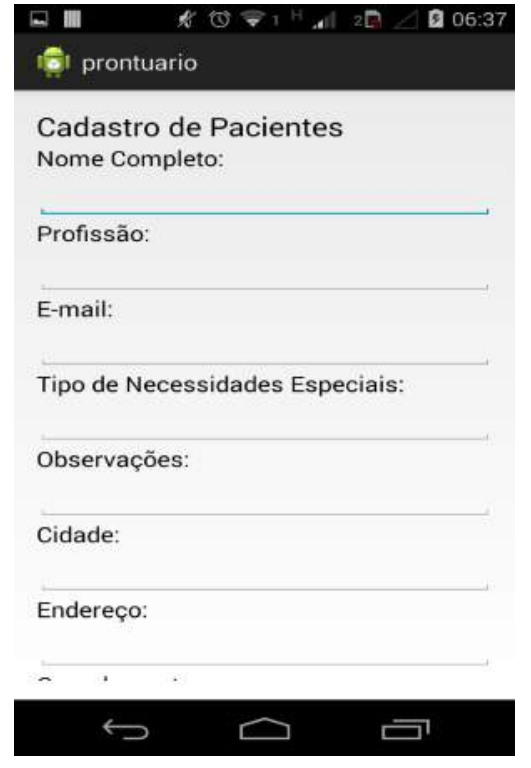

Figura 2: Campos de preenchimento de texto

dão e nível de dificuldade na fala. Caso o paciente apresente alguma dessas características, como mostrado na Figura 3, é permitida versatilidade ao operador da aplicação, que deverá sempre ser operada por um especialista a fim de manter a legitimidade dos dados coletados.

Apesar de unidos em uma mesma aplicação, as funcionalidades de cada parte manipulam seus dados separadamente. As funcionalidades de prontuário, como por exemplo, "Novo Cadastro" e "Dados Complementares", mantêm seus dados salvos em um banco de dados remoto e, as funcionalidades da ferramenta de análise como "Gravação", mantém os arquivos de vocalizações salvas localmente no dispositivo móvel em que a aplicação m-health estiver instalada, como está ilustrado na Figura 4.

Os dados que constituem o prontuário eletrônico de um paciente são salvos em um banco de dados remoto, afim de garantir que esses dados estejam disponíveis para não somente a solução m-health, mas também para outros sistemas que possam utilizar-se desses dados, de modo a compartilhar as informações. Tal característica possibilita atendimentos paralelos envolvendo diferentes especialistas com diferentes sistemas, no entanto tratando um mesmo paciente.

Os arquivos de vocalizações são salvas localmente no dispositivo móvel por sua relevância se estender primordialmente à aplicação m-health para o pré-diagnóstico. Somente o resultado do processamento da voz é encaminhado ao servidor remoto, uma vez que esta informação é que descreve a saúde da laringe e o arquivo digital do sinal amostrado é normalmente volumoso.

Para a descrição da saúde da laringe foi utilizado o período de pitch, avaliado utilizando o sinal da voz no domínio da frequência. O pitch, referente à frequência fundamental $\left(F_{0}\right)$, pode ser interpretado como um descritor da característica física da laringe, como por exemplo, pode diferir gêneros e faixas etárias.

Mulheres saudáveis, por exemplo, tendem a ter pregas vocais menores e menos espessas, o que às proporciona voz aguda, logo o pitch de suas vocalizações geralmente têm va- 


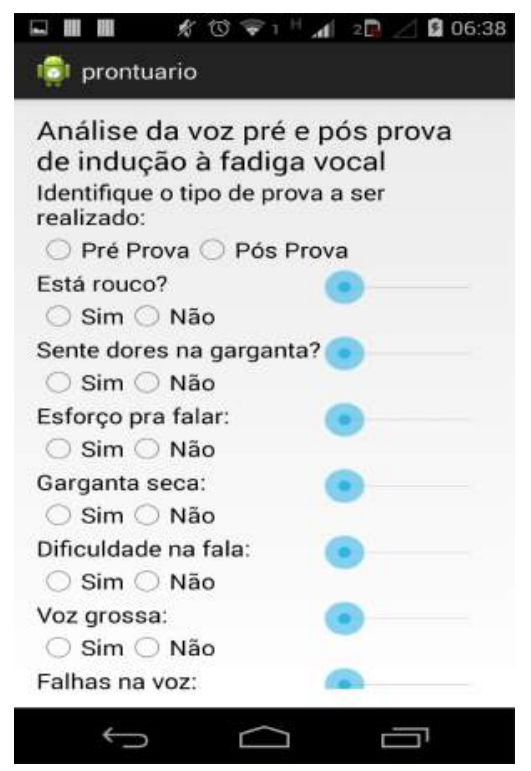

Figura 3: Campos de preenchimento com barras de rolagem

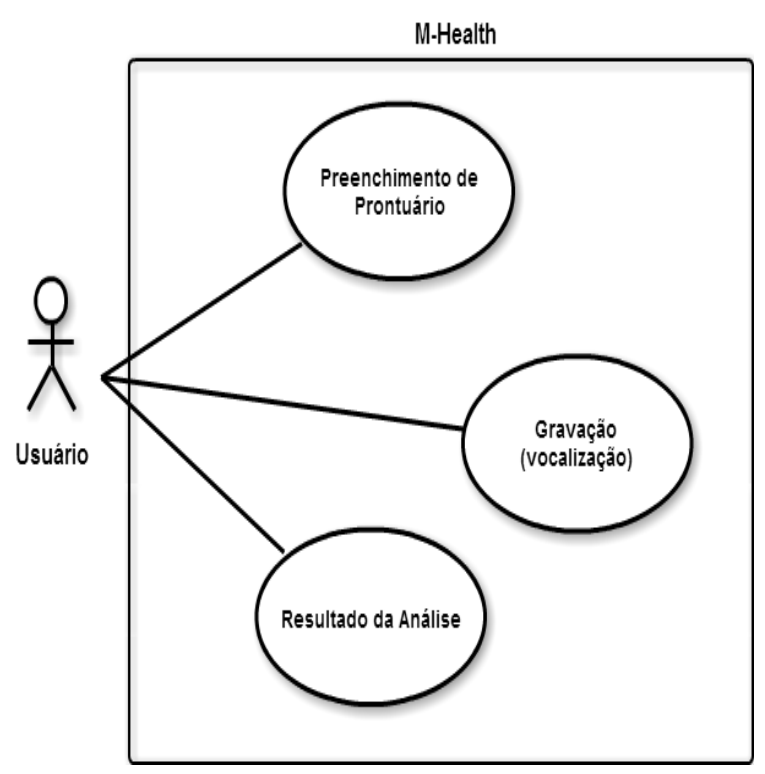

Figura 4: Diagrama da solução m-health lores específicos, variando entre 150 e $200 \mathrm{~Hz}$ [21]. Homens saudáveis tendem a ter a voz mais grave, logo frequências fundamentais de suas vocalizações possuem valores mais baixos quando comparado com o das mulheres, variando entre $88 \mathrm{~Hz}$ a $141 \mathrm{~Hz}$ [21]. Portanto, é possível a um especialista estabelecer por meio dos dados de um paciente, contidos no prontuário eletrônico, um intervalo de frequência fundamental que sua vocalização deveria alcançar. Assim, pode-se averiguar a saúde vocal dependendo dos valores de pitch obtidos pela ferramenta de análise, considerando os valores de normalidade de acordo com idade, gênero e hábitos do paciente. Por isso o conjunto de informações de voz e prontuário é necessário para compor a avaliação da saúde da laringe.

Como dito, o pitch é uma medida em frequência atribuída à sensação acústica da voz, que tem a propriedade de quantificar a frequência de voz de um indivíduo, ou seja, o quão grave ou agudo uma voz é [4]. A voz é adquirida no chamado domínio do tempo, isto é, as amostras são digitalizadas no decorrer da emissão pelo paciente. Para se calcular o valor do pitch em um sinal de voz no domínio do tempo, sinal temporal, é necessário o uso de técnicas de Processamento Digital de Sinais (PDS) para transformá-lo para o domínio da frequência. Entre as técnicas mais tradicionais temos as Transformadas Discretas de Fourier (Discrete Fourier Transform - DFT) e Wavelets (Discrete Wavelets Transform - DWT).

As DFT e DWT são calculadas computacionalmente por meio de algoritmos que possuem complexidade característica. A DFT está expressa na Equação 1, sendo $N$ o número de pontos do sinal temporal $x[\cdot]$ e $\omega$ a respectiva frequência angular. Existem algoritmos mais eficientes para o cômputo da DFT, conhecidos como algoritmos de Transformada Rápida de Fourier (Fast Fourier transform - FFT ) [4]. Essas alternativas, segundo [10], reduzem a ordem de complexidade computacional da mesma, de quadrática para logarítmica. O presente trabalho utiliza a implementação da FFT para análise das informações no domínio da frequência.

$$
X[\omega]=\sum_{n=0}^{N-1} x_{n} e^{\frac{-j 2 \pi n \omega}{N}}
$$

Sendo o m-health embarcado em um dispositivo de baixo poder computacional, a escolha pela FFT é necessária para o cálculo do pitch. Assim, a ferramenta m-health proposta, aliada a técnicas de PDS, detecta o pitch ou valor em $\mathrm{Hz}$ da frequência fundamental da vocalização de um paciente, Figura 5, propiciando ao especialista a observação e indicação de normalidade ou não do sinal da voz.

O reconhecimento de alterações na vocalização que podem refletir uma patologia na região laríngea, utilizando o pitch como parâmetro é uma abordagem computacionalmente menos complexa, com complexidade logarítmica e pouca exigência de memória. Desta forma, esta ferramenta pode ser utilizada em equipamentos com pouco poder de processamento, como smartphones, tablets e outros aparelhos portáteis, motivo pelo qual foi escolhido para integrar a solução m-Health proposta.

\section{MATERIAIS E MÉTODOS}

Ao considerar critérios acústicos, as patologias relacionadas à laringe são diferenciadas em relação a padrões perceptuais tais como rouquidão, aspereza e soprosidade [3, 7]. 


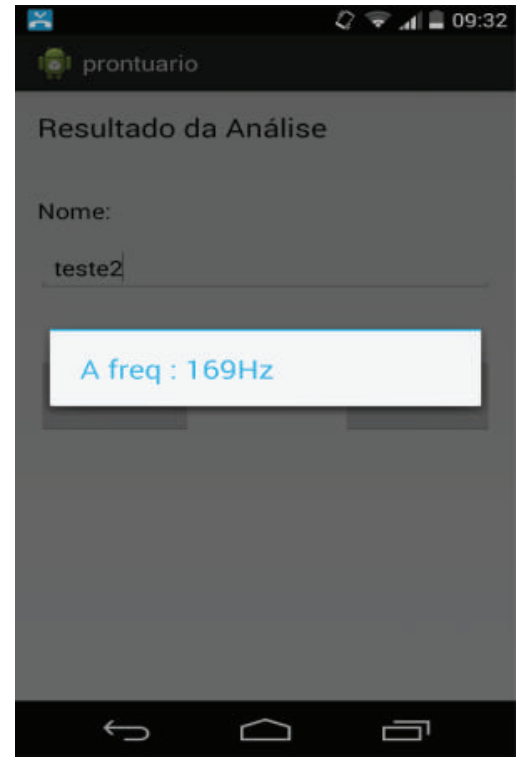

Figura 5: Resultado da análise da frequência fundamental

Desta forma, para avaliar a qualidade da solução proposta, foi utilizada uma base de dados real com sinais diagnosticados. A base de dados foi disponibilizada pela Universidade de São Paulo (USP) de Ribeirão Preto, aprovada pelo comitê de ética da mesma.

A base de dados continha fonemas do português brasileiro: $/ a /, / e /$ e $/ i /$. Porém, para os experimentos foi utilizado apenas o fonema $/ a /$, pois se sustentado em potências confortáveis ao locutor é considerado o melhor som vocálico para extração e medição dos parâmetros da voz [9]. Os sinais foram armazenados seguindo o padrão wave PCM [8] amostrando os sinais a $22050 \mathrm{~Hz}, 16$ bits e mono-canal. Cada voz amostrada digitalmente armazenou a sustenção do som vocálico por aproximadamente 5 segundos, em níveis confortáveis de potência, conforme um diálogo natural.

Para os experimentos foram utilizadas as vozes de dezenove indivíduos dos gêneros masculino e feminino. A escolha por indivíduos de gêneros diferentes se dá pela mudança da faixa do valor do pitch, que possibilita avaliar a solução de forma mais robusta.

A solução m-health proposta por esse trabalho, foi desenvolvida inteiramente para a plataforma móvel Android, devido a sua disponibilidade em uma grande variedade de dispositivos, além do baixo custo de desenvolvimento.

A sua criação ocorreu em duas etapas, a primeira se trata da criação de um prontuário eletrônico e a segunda, o desenvolvimento de uma ferramenta automatizada capaz de auxiliar a decisão médica em um pré-diagnóstico com base na quantificação da vocalização por meio do pitch.

Para os testes do m-health, o mesmo foi implantado em um dispositivo móvel Galaxy Tab 1, um tablet da empresa Samsung. Possui um processador ARM Cortex-A8 de 1 GHz, com 512 MB de memória RAM com versão do sistema operacional Android 2.2.

Para verificar o valor de pitch calculado pelo m-health, foi utilizado o software gratuito Audacity na versão 2.0.5 para sistema operacional Windows. O Audacity é um software para processamento de áudio e entre as ferramentas disponíveis apresenta a extração do valor do pitch. A escolha pelo Audacity se deu devido a sua ampla utilização por profissionais da área de áudio e por ser de uso gratuito.

A necessidade de comparação do valor calculado para o pitch entre um sistema conhecido e o m-Health é devido as adequações do algoritmo da FFT para o dispositivo móvel junto à avaliação da qualidade da aquisição do sinal sonoro.

\section{RESULTADOS E DISCUSSÕES}

Como proposto pelo trabalho, foram criadas interfaces para coletar os dados dos pacientes seguindo uma interface adequada para a quantificação da informação alimentada. Além de gravação de voz, as interfaces contém campos para preenchimento sobre hábitos e medidas do paciente em questão. Essas informações compõem o perfil do paciente e são importantes para uma análise mais completa e correlacionada com as características vocálicas do paciente.

Após a análise de vozes realizada pela solução proposta, sendo nove homens e dez mulheres, obteve-se $95 \%$ dos valores com diferença menor de $10 \mathrm{~Hz}$, como pode ser observado na Tabela 1. A diferença apresentada é aceita pois o sinal da sustentação da voz pode apresentar interferência do meio de aquisição, neste caso o microfone embutido do dispositivo utilizado.

Tabela 1: Comparação do valor do Pitch obtido

\begin{tabular}{|c|c|c|c|c|}
\hline Amostra & Audacity & m-health & Gênero & Diferença \\
\hline 1 & $151 \mathrm{~Hz}$ & $155 \mathrm{~Hz}$ & Feminino & $4 \mathrm{~Hz}$ \\
\hline 2 & $147 \mathrm{~Hz}$ & $147 \mathrm{~Hz}$ & Feminino & $0 \mathrm{~Hz}$ \\
\hline 3 & $197 \mathrm{~Hz}$ & $199 \mathrm{~Hz}$ & Feminino & $2 \mathrm{~Hz}$ \\
\hline 4 & $240 \mathrm{~Hz}$ & $251 \mathrm{~Hz}$ & Feminino & $11 \mathrm{~Hz}$ \\
\hline 5 & $235 \mathrm{~Hz}$ & $233 \mathrm{~Hz}$ & Feminino & $2 \mathrm{~Hz}$ \\
\hline 6 & $191 \mathrm{~Hz}$ & $190 \mathrm{~Hz}$ & Feminino & $1 \mathrm{~Hz}$ \\
\hline 7 & $233 \mathrm{~Hz}$ & $232 \mathrm{~Hz}$ & Feminino & $1 \mathrm{~Hz}$ \\
\hline 8 & $188 \mathrm{~Hz}$ & $181 \mathrm{~Hz}$ & Feminino & $7 \mathrm{~Hz}$ \\
\hline 9 & $226 \mathrm{~Hz}$ & $223 \mathrm{~Hz}$ & Feminino & $3 \mathrm{~Hz}$ \\
\hline 10 & $240 \mathrm{~Hz}$ & $241 \mathrm{~Hz}$ & Feminino & $1 \mathrm{~Hz}$ \\
\hline 11 & $172 \mathrm{~Hz}$ & $168 \mathrm{~Hz}$ & Masculino & $4 \mathrm{~Hz}$ \\
\hline 12 & $134 \mathrm{~Hz}$ & $136 \mathrm{~Hz}$ & Masculino & $2 \mathrm{~Hz}$ \\
\hline 13 & $137 \mathrm{~Hz}$ & $137 \mathrm{~Hz}$ & Masculino & $0 \mathrm{~Hz}$ \\
\hline 14 & $112 \mathrm{~Hz}$ & $119 \mathrm{~Hz}$ & Masculino & $7 \mathrm{~Hz}$ \\
\hline 15 & $117 \mathrm{~Hz}$ & $123 \mathrm{~Hz}$ & Masculino & $6 \mathrm{~Hz}$ \\
\hline 16 & $121 \mathrm{~Hz}$ & $129 \mathrm{~Hz}$ & Masculino & $8 \mathrm{~Hz}$ \\
\hline 17 & $110 \mathrm{~Hz}$ & $116 \mathrm{~Hz}$ & Masculino & $6 \mathrm{~Hz}$ \\
\hline 18 & $156 \mathrm{~Hz}$ & $162 \mathrm{~Hz}$ & Masculino & $6 \mathrm{~Hz}$ \\
\hline 19 & $118 \mathrm{~Hz}$ & $126 \mathrm{~Hz}$ & Masculino & $8 \mathrm{~Hz}$ \\
\hline
\end{tabular}

A maior diferença encontrada, $11 \mathrm{~Hz}$, foi para um sinal de voz do gênero feminino, no entanto, quando avaliada a distribuição estatística das diferenças entre m-health e Audacity, observamos que este resultado é um outlier no processo de extração do pitch.

Na Figura 6 é possível observar uma média de diferença de $2 \mathrm{~Hz}$ para vozes femininas e $6 \mathrm{~Hz}$ para as masculinas, ambos os gêneros apresentaram estabilidade semelhante. A média geral de diferença foi de aproximadamente $4 \mathrm{~Hz}$.

O processo de cálculo do pitch no dispositivo móvel teve duração semelhante ao Audacity, mesmo tendo hardware inferior. O tempo gasto para a execução da FFT para cada sinal de voz foi inferior à 1 segundo. 


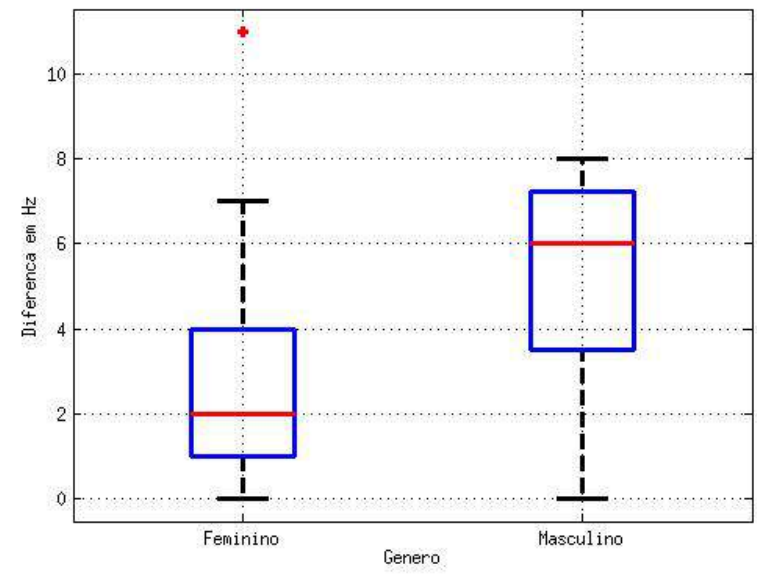

Figura 6: Diferença de valores obtidos para o pitch entre os gêneros feminino e masculino

Cabe ressaltar que qualquer afirmação relacionada a presença ou ausência de patologia não foi feita diretamente pelo desvio da faixa de normalidade do valor do pitch associada as informações do prontuário. Cabe ao especialista da área médica fazer tal avaliação.

\section{CONCLUSÃO}

Para realizar um diagnóstico preciso de patologias da região laríngea, um profissional da área da saúde necessita de informações atualizadas sobre o paciente, além de alguns exame que possam dar suporte ao seu diagnóstico. Atualmente os exames para diagnósticos de doenças na laringe são invasivos, de difícil realização e alto custo, além de desnecessários quando seu uso poderia ser descartado por um pré-diagnóstico.

A aplicação m-health produzida por este trabalho, integrou com sucesso um prontuário eletrônico com os dados médicos atualizados necessários para um atendimento, com uma ferramenta computacional automatizada que, ao analisar o sinal de voz de um paciente e propicia o auxílio do especialista na concepção de um pré-diagnóstico.

Os testes realizados em comparação com outra ferramenta já utilizada para a avaliação da voz mostrou que o m-health proposto tem resultado similar, com uma diferença de $4 \mathrm{~Hz}$ para o valor do pitch.

Para prosseguir com este projeto, pretende-se implantar módulos baseados em Aprendizado de Máquina para auxiliar o especialista no diagnóstico, indicando uma possível patologia presente na laringe.

\section{AGRADECIMENTOS}

Agradecemos à Universidade Estadual de Londrina e a Fundação Araucária pelas bolsas de Iniciação Científica. O projeto que resultou neste trabalho foi financiado pela Fundação Araucária - Chamada 04/2013 Programa Pesquisa para o Sistema Único de Saúde: Gestão Compartilhada em Saúde PPSUS Edição 2012.

\section{REFERÊNCIAS}

[1] J. E. Aikens, K. Zivin, R. Trivedi, and J. D. Piette. Diabetes self-management support using mhealth and enhanced informal caregiving. Journal of diabetes and its complications, 28(2):171-176, 2014.

[2] O. A. Alsos, A. Das, and D. Svanæs. Mobile health it: The effect of user interface and form factor on doctor-patient communication. International journal of medical informatics, 81(1):12-28, 2012.

[3] R. J. Baken and R. F. Orlikoff. Clinical measurement of speech and voice. Cengage Learning, 2000.

[4] S. Barbon Júnior. Identificação de patologias na laringe com base na Discriminative Paraconsistent Machine (DPM). PhD thesis, Universidade de São Paulo, 2011.

[5] C. G. Bayne and P. A. Boling. New diagnostic and information technology for mobile medical care. Clinics in geriatric medicine, 25(1):93-107, 2009.

[6] A. M. Benavides, R. F. Pozo, D. T. Toledano, J. L. B. Murillo, E. L. Gonzalo, and L. H. Gómez. Analysis of voice features related to obstructive sleep apnoea and their application in diagnosis support. Computer Speech \&6 Language, 28(2):434-452, 2014.

[7] D. Bless. Measurement of vocal function. Otolaryngologic Clinics of North America, 24(5):1023-1033, 1991.

[8] M. Bosi and R. E. Goldberg. Introduction to digital audio coding and standards. Springer Science \& Business Media, 2003.

[9] M. de Oliveira Rosa, J. C. Pereira, and M. Grellet. Adaptive estimation of residue signal for voice pathology diagnosis. Biomedical Engineering, IEEE Transactions on, 47(1):96-104, 2000.

[10] J. R. Deller, J. G. Proakis, and J. H. Hansen. Discrete-time processing of speech signals. IEEE New York, NY, USA:, 2000.

[11] K. W. dos Santos, C. S. Trindade, and V. D. C. G. M. Fernandes, Renada A. Utilização de softwares em pesquisas científicas de fonoaudiologia. Journal of Health Informatics, 4(2):55-8, 2012.

[12] J. A. Gómez-Puerta, A. Cisternas, M. V. Hernández, V. Ruiz-Esquide, I. Vilaseca, and R. Sanmartí. Laryngeal assessment by videolaryngostroboscopy in patients with rheumatoid arthritis. Reumatología Clinica (English Edition), 10(1):32-36, 2014.

[13] S. Jenal and Y. D. M. Évora. Desafio da implantação do prontuário eletrônico do paciente. Journal of Health Informatics, 4(Número Especial - SIIENF 2012):206-9, 2012.

[14] S. Jenal and Y. D. M. Évora. Revisão de literatura: Implantação de prontuário eletrônico do paciente. Journal of Health Informatics, 4(4):176-81, 2012.

[15] L. Jennings, A. Omoni, A. Akerele, Y. Ibrahim, and E. Ekanem. Disparities in mobile phone access and maternal health service utilization in nigeria: a population-based survey. International Journal of Medical Informatics, 2015.

[16] S. A. Marques, H. M. A. Ricz, and L. N. Aguiar-Ricz. Analysis of the magnitude of the symptoms of vocal fatigue pre and post test of continuous speech. 16:(Suppl. 1):24, 2012.

[17] L. Namorato, A. J. Cavalcanti Neto, F. V. Garani, 
P. O. Braga, and S. A. S. Lustosa. A utilização do prontuário eletrônico do paciente por médicos do hospital municipal dr. munir rafful: um estudo de caso. Journal of Health Informatics, 5(2):39-43, 2013.

[18] A. Pompilio Junior and E. Ermetice. Indicadores de uso do prontuário eletrônico do paciente. Journal of Health Informatics, 3(1):9-12, 2011.

[19] K. P. Rothfield and S. G. Russo. Videolaryngoscopy: should it replace direct laryngoscopy? a pro-con debate. Journal of clinical anesthesia, 24(7):593-597, 2012.

[20] E. Y. Saito, G. V. E. Rojas, L. N. A. Ricz, and S. Barbon Jr. Reconhecimento automático de distonia laríngea com base na sustentação do pitch. Journal of Health Informatics, 6(2):53-9, 2014.

[21] R. M. Silva, S. G. Mazucato, and S. Barbon Jr. Estudo sobre os parâmetros acústicos relevantes para a verificaçao de patologias associadasa voz. In Anais do Congresso de Matemática Aplicada e Computacional CMAC Sudeste, pages 282-285. SBC, 2011.

[22] Y. Wang, M. Chen, Z. Tao, Q. Hua, S. Chen, and B. Xiao. Identification of predictive biomarkers for early diagnosis of larynx carcinoma based on microrna expression data. Cancer genetics, 206(9):340-346, 2013. 
XI Brazilian Symposium on Information System, Goiânia, GO, May 26-29, 2015. 